\title{
L'analyse sociétale des relations entre système éducatif et système productif
}

Comparaison France - Allemagne - Japon

Marc Maurice

\section{OpenEdition}

\section{Journals}

Édition électronique

URL : http://journals.openedition.org/ries/4299

DOI : $10.4000 /$ ries.4299

ISSN : 2261-4265

Éditeur

Centre international d'études pédagogiques

Édition imprimée

Date de publication : 1 mars 1994

Pagination : $35-45$

ISSN : 1254-4590

Référence électronique

Marc Maurice, "L'analyse sociétale des relations entre système éducatif et système productif », Revue internationale d'éducation de Sèvres [En ligne], 01 | 1994, mis en ligne le 20 avril 2015, consulté le 23 avril 2019. URL : http://journals.openedition.org/ries/4299; DOI : 10.4000/ries.4299

Ce document a été généré automatiquement le 23 avril 2019

(c) Tous droits réservés 


\title{
L'analyse sociétale des relations entre système éducatif et système productif
}

\author{
Comparaison France - Allemagne - Japon
}

\section{Marc Maurice}

1 La question des relations entre système éducatif et système productif, ou entre l'école et l'entreprise n'est certes pas nouvelle ; on la retrouve tout au long de l'histoire, des débuts de l'industrialisation jusqu'à nos jours. Elle se pose cependant aujourd'hui en des termes nouveaux, et les réponses qu'on peut lui apporter constituent autant d'enjeux, dans la conjoncture présente, face à la montée du chômage et du retour escompté de la croissance.

2 Ce n'est sans doute pas un hasard si, dans un tel contexte, on fait appel dans les débats qu'il suscite au « modèle allemand » ou au « modèle japonais ». Le premier est chargé des vertus de son système éducatif et de formation ; le second attire l'attention par l'efficacité du système de travail de ses entreprises.

\section{Les rapports entre l'école et l'entreprise}

3 Mais, si comparaison internationale il y a, cette double référence, allemande et japonaise, ne traduit-elle pas aussi la tendance, qui n'est d'ailleurs pas que française, à traiter séparément les problèmes de l'éducation ou de formation et les problèmes de l'organisation du travail comme si on avait à faire à deux entités séparées - l'école et l'entreprise - mises simplement en relation par le jeu de l'offre et de la demande?

4 Sans doute une telle formulation est-elle quelque peu provocante, alors que des efforts sont faits en France depuis peu pour rapprocher les établissements scolaires et les entreprises, et que des innovations se font jour pour développer des formations en alternance ou des "partenariats » entre des institutions qui tendaient jusqu'ici à s’ignorer ou à se méconnaître. 
5 Ne retrouverait-on pas d'ailleurs une tendance analogue parmi les spécialistes de l'éducation et ceux du travail ou des entreprises, qu'ils soient économistes ou sociologues? Il est vrai que les théories qui prennent en compte l'éducation ou l'entreprise n'ont que rarement eu la capacité de traiter les interdépendances complexes entre ces différents champs sociaux, qu'il s'agisse de la théorie du capital humain ou de celle de la reproduction sociale. Dans un cas, l'éducation est traitée comme pur investissement sans que l'on s'interroge sur les relations entre formes d'acquisition des savoirs et formes d'organisation du travail ; dans l'autre celles-ci sont traitées de façon quelque peu simplifiée, comme si les dernières ne faisaient que redoubler les effets des premières.

6 Ne faut-il pas dès lors considérer les rapports école-entreprise comme construit social et donc objet d'analyse?

\section{L'analyse sociétale : une nouvelle façon de traiter des relations école-entreprise}

7 L'analyse sociétale, développée par une équipe de chercheurs du LEST ${ }^{1}$, à partir de comparaisons internationales entre des entreprises françaises, allemandes et japonaises permet de traiter les rapports entre système éducatif et système productif, dans la mesure où elle considère que ni l'entreprise, ni l'école ne sont des entités isolables, participant l'une et l'autre, et dans leurs relations, au développement de la société.

8 Autrement dit, chaque société, à travers son histoire et à partir des pratiques de ses acteurs, construit des formes d'interdépendances entre l'école et l'entreprise, entre les «faits de socialisation» et les "faits d'organisation ». Se constituent dès lors entre le système éducatif et le système productif un ensemble de "rapports sociaux » qui contribuent à la construction sociale des acteurs, qu'il s'agisse - on le verra - de l'ouvrier qualifié, du contremaître ou de l'ingénieur, et des relations qu'ils entretiennent entre eux.

9 Ce type d'approche, et les comparaisons internationales qu'il a pu orienter, permet aussi bien de dépasser (de manière critique) les clivages, voire les oppositions, entre éducation, emploi et travail. Il fournit en outre une aide conceptuelle pour l'analyse des rapports dont on prend conscience progressivement aujourd'hui - entre l'école et l'entreprise, mais que l'on traite trop souvent dans une perspective purement institutionnelle. L'analyse sociétale permet enfin de mesurer ce que l'on peut attendre des comparaisons internationales, et des références aux « modèles », allemand et japonais par exemple.

10 Sans pouvoir ici développer ces différentes questions, on illustrera plutôt la démarche de l'analyse sociétale par la manière dont elle traite des rapports entre "faits de socialisation » et « faits d'organisation », dans des contextes nationaux différents ${ }^{2}$.

11 On comparera d'abord la situation française et allemande, en tenant compte des évolutions récentes de ces deux pays. On introduira ensuite le cas du Japon, dont le contraste avec deux sociétés occidentales permet de mieux comprendre ce qui peut rapprocher ou au contraire diversifier chacun de ces pays.

12 Sans détailler ici les caractéristiques institutionnelles et économiques de chaque pays, ni l'histoire de leurs systèmes éducatif et productif, on soulignera plutôt la logique sociale qui sous-tend les relations école-entreprise, en se centrant plus particulièrement sur la 
formation professionnelle ; bien que celle-ci ne soit pas, en fait, isolable de la formation générale au sein de chaque système éducatif.

\section{Les leçons de la comparaison France-Allemagne}

13 Si dans chaque pays le système éducatif comprend en effet un enseignement général et une formation professionnelle, l'importance accordée à l'un ou l'autre, et surtout les modalités de mise en œuvre peuvent varier d'un pays à l'autre et même au cours de l'histoire d'un même pays. En ce sens, le système éducatif apparaît bien comme un « construit social» dont on verra qu'il entre en composition avec cet autre « construit social » qu'est l'entreprise ou le système productif. Ni l'un, ni l'autre ne sont jamais tout à fait stabilisés, même si on peut leur reconnaitre dans le temps une certaine inertie.

\section{La formation professionnelle en France et en Allemagne (ex RFA) ${ }^{3}$}

Une vue cavalière de l'histoire du système éducatif français met en évidence l'importance accordée dans ce pays à la formation générale par rapport à la formation professionnelle, même si celle-ci a pu fluctuer selon les périodes. La crise économique, dont on a du mal à sortir, a fait prendre conscience une fois encore, au cours de la dernière décennie, de cette «faiblesse» endémique de la société française. Des mesures diverses (souvent d'ordre politique) ont été prises et continuent de l'être pour tenter de renforcer la formation professionnelle, des ouvriers notamment, en s'inspirant du système allemand de formation en alternance ${ }^{4}$.

Considéré globalement, le système éducatif français est constitué selon une «logique de niveaux combinant dans une même hiérarchie formations générale, technique et supérieure $»^{5}$. Mais la " voie royale » reste bien encore celle qui, partant du baccalauréat, conduit à l'université ou aux grandes écoles d'ingénieurs. En dépit d'une certaine "démocratisation" de certains segments du système éducatif, sous la poussée démographique et des besoins de l'économie, ce système repose toujours pour l'essentiel sur le principe de l'extraction des élites. Hormis certaines formations d'ingénieurs (dont les plus prestigieuses sont en fait plutôt des formations générales de haut niveau), la formation professionnelle et technique a été considérée pendant longtemps (et le reste encore en partie) comme résiduelle, ou comme une voie d'échec, sans être adaptée aux besoins des entreprises.

efforts récents et relativement importants qui ont été faits par les gouvernements successifs depuis le début des années 80 tendent à valoriser davantage la formation professionnelle et à la rapprocher des besoins de l'économie, sans modifier pour autant les formes de division du travail ou de hiérarchisation de l'organisation: tout se passe comme si la valorisation de la formation professionnelle avait pour effet de créer de nouvelles strates (correspondant à de nouveaux diplômes, ou à de nouvelles filières qu'il s'agisse des ouvriers, des techniciens, ou des ingénieurs ${ }^{6}$.

17 Le système allemand fonctionne selon une logique bien différente. Dans ce cas la formation professionnelle se positionne de façon différente par rapport à la formation générale. L'une et l'autre apparaissent à la fois distinctes et complémentaires, plutôt que hiérarchisées comme dans le système français. Chacune a ses propres exigences sans être pour autant cloisonnées l'une par rapport à l'autre. Des liens existent entre formation 
initiale et formation continue, ce qui permet tout au long de la carrière des passages entre formation générale et formation professionnelle. Ce système tout en étant relativement flexible n'en demeure pas moins très contrôlé par les diplômes qui contribuent à leur tour, on le verra, à contrôler les promotions et les mobilités au sein de l'entreprise.

Mais sans entrer ici dans le détail des filières respectives, rappelons brièvement les caractéristiques du «système dual» de formation (ou formation en alternance) dont le socle est constitué par l'apprentissage. Là se situe en effet ce qui différencie le plus le système allemand du système français, lorsqu'on compare comment se répartit dans chaque pays la population active par niveau de diplôme. Ainsi, selon une enquête récente, en 1987, 72 \% des sortants du système scolaire général avaient effectué une formation "duale ", directement ou en formation continue quelques années après leur sortie de l'école ${ }^{7}$. De même, parmi les actifs occupés, 55,4 \% des Allemands en 1989 avaient acquis un brevet dual (diplôme d'apprentissage) ou un diplôme de niveau équivalent. Seulement $28,9 \%$ des Français avaient un diplôme analogue (CAP, BEP), mais acquis dans des conditions bien différentes ${ }^{8}$.

On peut estimer qu'en Allemagne la formation professionnelle initiale est avant tout une formation en entreprise. Celle-ci est réglée par un contrat entre l'employeur et l'apprenti, et s'effectue sous la tutelle d'un Meister dont la fonction pédagogique est d'ailleurs socialement légitimée au-delà de sa reconnaissance par l'entreprise 9 .

À cela s'ajoute l'importance de la formation continue qui se développe à partir de la base commune de l'apprentissage. La formation d'ouvrier qualifié (Facharbeiter) constitue pratiquement le point de passage obligé de la majorité du personnel technique des entreprises, y compris d'une partie des ingénieurs. Rappelons ici que deux voies conduisent en effet aux diplômes d'ingénieur: celle des écoles techniques supérieures (Fachhochschulen) que l'on vient d'évoquer et celle des universités techniques (Technische Universität ou Technische Hochschulen) dont les études sont plus longues et plus théoriques. Mais il s'agit en fait de deux formations complémentaires que l'entreprise gère comme telles sans nécessairement les hiérarchiser. Ceci s'inscrit dans la logique d'un système fondé sur la complémentarité des compétences (à la fois pratiques et théoriques) plutôt que sur la hiérarchie des positions, et dans lequel les salariés ont des possibilités, par formation continue sanctionnée par des diplômes spécifiques, d'évoluer sans être prisonniers de leur filière d'origine.

\section{Les interdépendances entre l'école et l'entreprise : le rôle de l'entreprise en France et en Allemagne}

Les différences qui se dégagent de cette rapide présentation ne constituent en fait qu'une partie du tableau : la formation professionnelle, quel que soit son contenu, ne prend toute son efficacité que dans l'usage qu'en font les entreprises. Mais il faut ajouter que l'entreprise peut directement ou indirectement influer sur la qualité de la formation professionnelle telle qu'elle est conçue et organisée par le système éducatif. Ce qu'illustre bien le système allemand.

Dans ce dernier cas, le socle de l'apprentissage «dual » permet de fonder, ce que nous avons appelé dans nos recherches un espace de qualification composé par les ouvriers qualifiés, les Meister, et les ingénieurs (issus des Fachhochschule ${ }^{10}$ ). Ces différentes 
catégories partagent en effet une commune référence d'apprentissage pratique et théorique sur laquelle peuvent se développer des formes de coopération entre eux.

Mais la façon dont se constituent cet espace de qualification et les acteurs qui le structurent est tout autant liée aux formes d'organisation du travail et de gestion mises en œuvre par les entreprises elles-mêmes. Tout se passe comme si les entreprises légitimaient par leurs pratiques les diplômes qui sanctionnent les compétences des différentes catégories d'acteurs que l'on vient d'évoquer.

Ceci se vérifie d'autant plus que les entreprises participent, pour une large partie, à l'organisation de la formation professionnelle de base dont elles financent plus des deux tiers. En ce sens la formation apparaît bien comme un investissement à plusieurs titres ${ }^{11}$.

Le système de travail observé dans des entreprises françaises comparables aux entreprises allemandes devait mettre en évidence des logiques différentes. Rappelons-en brièvement quelques traits significatifs ${ }^{12}$. L'accès aux emplois est dans ce cas moins contrôlé par les diplômes qu'en Allemagne, et l'acquisition du diplôme lui-même est plus indépendant de l'entreprise et de ses pratiques; bien que l'on puisse observer plus récemment, à partir de nouvelles procédures, impulsées d'ailleurs par les pouvoirs publics, une plus forte implication des entreprises dans certaines formations.

Il en résulte, dans les cas encore les plus fréquents, une moindre adéquation entre la qualification acquise et le contenu de l'emploi occupé ; l'entreprise française accordant une certaine importance à l'ancienneté dans sa gestion des "ressources humaines"; comme si, qu'ils soient diplômés ou pas, les salariés devaient être "socialisés " à ses normes ou à sa «culture $»^{13}$. Ceci entraîne des formes de mobilité interne et de montée dans la hiérarchie, contrôlées plus par l'ancienneté ou l'expérience que par le diplôme ; ce qui pourrait rapprocher - formellement du moins - sur ce point les entreprises françaises des entreprises japonaises.

Pour résumer, dans le cas de la France, deux logiques semblent se renforcer : celle de l'extraction des élites dans le système éducatif, ce qui va de pair avec une formation professionnelle qui a été pendant longtemps plutôt réservée aux exclus de la «voie royale ", et celle de l'attraction hiérarchique qui a eu pour effet de dévaloriser les fonctions technico-professionnelles attachées à la production, secteur lui-même moins prestigieux que d'autres.

On reviendra en conclusion sur les évolutions que peuvent connaître aussi bien le système allemand que le système français, dont on a présenté jusqu'ici une image relativement statique. Ces deux systèmes sont bien entendu dynamiques et évolutifs; ce qui ne signifie pas pour autant qu'ils ne soient pas conditionnés par les formes sociales qui ont contribué jusqu'ici à les construire.

\section{Comment le Japon conçoit-il la relation système éducatif et système productif ? Et quel est le rôle de l'entreprise dans cette relation?}

On rappellera brièvement quelques traits du système éducatif japonais, en insistant surtout sur le rôle de l'entreprise dans la formation professionnelle de l'ensemble des salariés, car la comparaison avec le Japon apporte à cet égard des éléments de réflexion nouveaux par rapport à ceux que révèle la comparaison France-Allemagne. 


\section{Le système éducatif japonais}

à celle de la France, si l'on s'en tient à la structure d'ensemble du système éducatif, en particulier à l'importance accordée à la formation générale et à la faiblesse relative de l'enseignement technique.

etracera pas ici l'historique du système éducatif japonais qui a connu des évolutions sensibles en particulier après la dernière guerre mondiale, notamment sous l'influence américaine de "démocratisation" de la société japonaise ${ }^{14}$. Rappelons seulement ici que le système éducatif japonais se caractérise aujourd'hui par un enseignement supérieur de masse et par l'importance qu'y tiennent les établissements privés $^{15}$. Le taux d'accès à l'enseignement supérieur a connu un développement très important durant la période de croissance, passant de 10,3\% en 1960 à $38 \%$ en 1980 . L'attraction que représentent les universités et l'enseignement secondaire (niveau baccalauréat), traduit l'importance croissante accordée à la formation générale, au détriment de la formation professionnelle ou technique scolarisée ${ }^{16}$.

Ainsi, depuis les années 60 et 70 on observe une désaffection continue pour ces enseignements, au profit de ce qui apparaît de plus en plus comme la « voie royale » : le secondaire général, puis l'accès (par concours) aux universités (cursus en deux ans, quatre ans ou plus). La compétition est d'ailleurs forte à ces deux niveaux et l'on s'y prépare souvent en suivant des cours spécialisés dans des écoles privées (juku).

Ces enseignements généraux fournissent les connaissances de base à partir desquelles les entreprises (ou les plus grandes d'entre elles) pourront développer les formations professionnelles correspondant à leurs besoins. Dans l'ensemble, on s'accorde d'ailleurs à reconnaitre le caractère relativement académique des enseignements aussi bien dans le secondaire que dans le supérieur.

Tout se passe comme si, surtout à partir des années 60-70, un partage des rôles s'était peu à peu établi entre l'école et l'entreprise, entre le système éducatif à qui revient la formation générale et les entreprises qui de facto prennent en charge l'essentiel de la formation professionnelle. Ceci ne doit pas pour autant faire tenir pour négligeables les enseignements techniques déjà évoqués ${ }^{17}$. Ils dispensent des formations théoriques de base, à chaque niveau (secondaire et supérieur), que les entreprises utilisent comme autant de « signaux » de potentialités lors de leur recrutement.

D’une façon générale, la hiérarchisation des établissements privés ou publics permet aux entreprises d'effectuer une première sélection; ce que facilitent d'ailleurs les réseaux de professeurs dans les lycées comme dans les universités, qui recommandent leurs meilleurs candidats auprès des entreprises avec lesquelles ils sont en relations suivies.

D'ailleurs, les grandes entreprises japonaises qui investissent le plus dans la formation ont tendance à accorder, lors des recrutements, une préférence aux jeunes qui n'ont pas reçu une formation trop spécialisée. Les qualités premières qui sont recherchées sont leur capacité d'adaptation sociale autant que professionnelle, et un large « potentiel ».

Revue internationale d'éducation de Sèvres, 01 | 1994 


\section{L'entreprise japonaise comme organisation qualifiante (learning organization)} favorable à la coopération. De même la hiérarchie des positions se fonde davantage sur l'expérience acquise, notamment par des mobilités horizontales, que par la montée dans l'échelle des statuts. Celle-ci est plutôt la résultante de celle-là, et non l'inverse ${ }^{19}$. Ce qui conforte encore la notion d'apprentissage collectif qui sous-tend les formes d'organisation et de fonctionnement de l'entreprise japonaise. 

système, quitte à transformer celui-ci pour partie. Il ne s'agit pas dès lors d'imiter le "modèle" allemand ou japonais; emprunter, c'est dans ce cas se mettre plutôt en position d'apprentissage et d'innovation.

\section{BIBLIOGRAPHIE}

AOKI M. (1991), Economie japonaise : information, motivations et marchandage, Paris, Economica, 1991. 
CEREQ (1992), Le système de formation professionnelle en Allemagne, Collection des études, $\mathrm{n}$ -61, février.

MAURICE M., SELLIER F., SILVESTRE J.-J. (1982), Politique d'éducation et organisation industrielle en France et en Allemagne, PUF, Paris. (Traduction : The Social Foundations of Industrial Power, MIT Press, Boston, 1986).

MAURICE M., MANNARI H, TAKEOKA Y., INOKI T. (1988), Des entreprises françaises et japonaises face à la mécatronique : acteurs et organisation de la dynamique industrielle, LEST-CNRS, Aix-enProvence (rapport de recherche).

MOBUS M., SEVESTRE P. (1991), «Formation professionnelle et emploi : un lien plus marqué en Allemagne ", Économie et statistique, $n^{\circ}$ 246-247, septembre-octobre.

NOHARA H. (1983), Enseignement technique, qualification industrielle et marché du travail : le cas du Japon, note de travail, LEST-CRNS.

PLANTIER J. (1989), Technique et société au Japon. Histoire sociale de l'enseignement technique (1945-1985), Paris, L'Harmattan.

\section{NOTES}

1. Laboratoire d'économie et de sociologie du travail.

2. Notons ici que notre démarche ne se réduit pas à donner priorité aux "faits de socialisation ", comme on a pu le penser parfois. Elle se centre au contraire sur les interdépendances entre ces différentes dimensions, et sur les médiations qu'elles impliquent. Cf. R. Boyer et E. Cazoli, «Changement de paradigme productif et rapport éducatif: performances de croissance comparées France-Allemagne", p. 20, in Changement des organisations, Conventions, Régulations, INRA, École-Chercheurs, Giens, 27-30 septembre 1993.

3. On ne traitera pas ici de l'Allemagne réunifiée, dans la mesure où l'on n'a pas suffisamment de recul pour le faire à partir de données fiables.

4. Ce qui suscite des débats, déjà anciens, à propos du « monopole de l'éducation nationale » de la part de certains syndicats d'enseignement.

5. Selon la formule de Martine Mobùs et Patrick Sevestre (1991), « Formation professionnelle et emploi : un lien plus marqué en Allemagne ", in Économie et Statistique, n 246-247, article dont nous nous inspirons d'ailleurs dans cette partie.

6. L'exemple des "ingénieurs technologues" issus du rapport Descomps est illustrant de ce phénomène: cette nouvelle catégorie d'ingénieurs par formation interne de techniciens va constituer une strate intermédiaire entre les techniciens supérieurs et les autres catégories d'ingénieurs issus d'écoles elles-mêmes hiérarchisées en fonction de leur degré de proximité avec les grandes écoles.

7. Cf. M. Mobüs et P. Sevestre, op. cit.

8. Rappelons que $19,5 \%$ des actifs allemands n'avaient pas reçu de formation professionnelle en 1987 , contre $43,1 \%$ des actifs français.

9. En 1985, par exemple, 1400000 actifs possédaient le diplôme de Meister, dans une population de 29 millions d'actifs

10. Appelés aussi antérieurement « ingénieurs gradués».

11. B. Lutz, «Les principes de fonctionnement du système dual : la régulation par le marché », in collection les Études, n 61, CEREQ, février 1992.

12. Cf. M. Maurice, F. Sellier, J.-J. Silvestre, Politique d'éducation et organisation industrielle en France et en Allemagne, PUF, Paris, 1982. 
13. Un tel système pourrait être qualifié d'autodidacte, et ce n'est pas un hasard si l'on trouve encore dans les entreprises françaises les plus évoluées techniquement une proportion relativement importante des cadres «autodidactes", c'est-à-dire n'ayant pas de diplômes d'enseignement supérieur.

14. Notons seulement que cette influence a plutôt renforcé dans la réforme de 1947 l'enseignement général et en intégrant les enseignements techniques dans la filière du secondaire, ceux-ci ont été dévalorisés en tant que tels. Cf. Joëlle Plantier, Technique et société au Japon : Histoire sociale de l'enseignement technique (1945-1985), Paris, 1989.

15. $1,2 \%$ pour l'éducation obligatoire, $28 \%$ dans le secondaire, $77 \%$ dans le supérieur.

16. Bien que le Japon ait développé l'ensemble des filières de l'enseignement technique aux différents cycles du système éducatif.

17. Notons, entre autres, les collèges techniques auxquels on accède après le premier cycle des études secondaires, et qui forment en cinq ans des «techniciens». Cette formation permet d'accéder au cycle long des universités. En 1989, près de 52000 jeunes suivaient ces cours, dispensés dans 62 collèges. Même si on y ajoute certains enseignements des écoles de formation spéciales, on est loin des 450000 étudiants qui, la même année, suivaient les cours des universités en science et engineering.

18. Faut-il noter que les généralisations utilisées dans cet article s'agissant des entreprises françaises, allemandes ou japonaises traduisent, bien entendu, des "types idéaux ", bien que certains de leurs traits ressortent aussi des enquêtes précises que nous avons menées dans ces trois pays au cours de la dernière décennie. On ne peut que renvoyer le lecteur aux rapports de recherche, publiés par le LEST.

19. Ceci se vérifie aussi parmi les dirigeants dont la majorité dans les entreprises l'est devenu après une longue expérience dans l'entreprise.

20. On distinguait dans la comparaison France-Allemagne trois types de « rapports sociaux»: rapport éducatif, rapport organisationnel, rapport industriel (ce dernier traduisant des formes de régulation sociale entre l'entreprise, l'État et les salariés).

\section{RÉSUMÉS}

L'auteur expose les points forts de l'approche sociétale, une nouvelle façon de traiter des relations école-entreprise à partir d'une comparaison des cas français, allemand et japonais.

\section{INDEX}

Index géographique : France, Allemagne, Japon

Mots-clés : éducation comparée, politique éducative, relation école-entreprise, système éducatif, système social 


\section{AUTEUR}

MARC MAURICE

Laboratoire d'économie et de sociologie du travail (LEST), Aix-en-Provence. 